\title{
NIGHT IMAGE ENHANCEMENT USING FUSION TECHNIQUE
}

\author{
Amritpal Singh ${ }^{1}$, Vijay Kumar Banga ${ }^{2}$ \\ ${ }^{1}$ Amritpal Singh, ${ }^{2}$ Dr. Vijay Kumar Banga, E.C.E Department, A.C. E.T, Amritsar, Punjab, India \\ apspunn@gmail.com, vijaykumar.banga@gmail.com
}

\begin{abstract}
Video surveillance is used in monitoring the road traffic. However because the surveillance system is limited by many objective factors. Surveillance video may not be seen clearly. Especially under the weak light conditions, the picture quality of the night video is very poor.

In this paper, we propose an enhancement method of nighttime images for surveillance camera. We apply the moving target extraction technology and illumination estimation theory and combine the nighttime image with the daytime background by the image fusion. The proposed method recoveries scene information of the night video and highlights the details. The resultant images show the vehicles of the night time but surrounding is fused from the day image taken at day. In this, it is observed that the PSNR value is 65.9 $d B$ is very high and MSE is 0.128 which is very low as compared with earlier reported night image enhancement technique [1]. Experimental results prove that our method is effective.
\end{abstract}

Index Terms: Image enhancement, Motion detection, Image fusion

\section{INTRODUCTION}

Night video enhancement is one of the most important and difficult component of the video security surveillance system. Most images taken from scenes with non uniform distributed illumination show the problem of being too contrasty. The images then can be divided into several different regions according to their need for enhancement [2]. Until recently a grey- or green scale representation of night vision imagery has been the standard. However, the increasing availability of fused and multiband infrared and visual night vision systems has led to a growing interest in the color display of night vision imagery [3, 4, 5, 6 and 7]. People have difficulty in understanding nighttime video because of the following reasons: Firstly, due to reasons of sensor noises or low luminance, night images appear much noise. Secondly, the brightness distributes unevenly because of the factitious illumination at night. In view of the above problems, the research on the video enhancement technology at night is meaningful. Nowadays, many techniques for image enhancement are discussed, such as contrast stretching, slicing, histogram equalization etc. The enhancement results of the traditional algorithm are not ideal for the nighttime video; these algorithms may cause excessive exposure or amplify noise. Therefore, enhancement algorithms combined with daytime image have attracted attention of many researchers $[8,9,10]$.

In this paper, we propose an enhancement method of nighttime images for a surveillance camera. We not only apply the moving target extraction technology, Retinex theory and fusion method, but also add an index mapping function to the fusion image. The objective of our method guarantees that most of the important contexts in the scene are synthesized to create a much clearer video for observers.

We present a novel technique for night image enhancement technique which overcomes very low intensity images to a good intensity image, in order to give better visibility to the inspectors inspecting the highways. This night image enhancement technique is based on hybrid model of day and night images for the same area of that highway which is under surveillance. This technique applies the hybrid image to increase the contrast of local part of the image which is stable (not moving objects) in the night images to that of non-stable (moving objects) on the highway [11].

\section{PROPOSED METHOD}

The objective of the proposed method is to increase the intensity of the night time images and to provide for information about the captured area and increase the situational awareness. The proposed method is computationally fast and simple. 


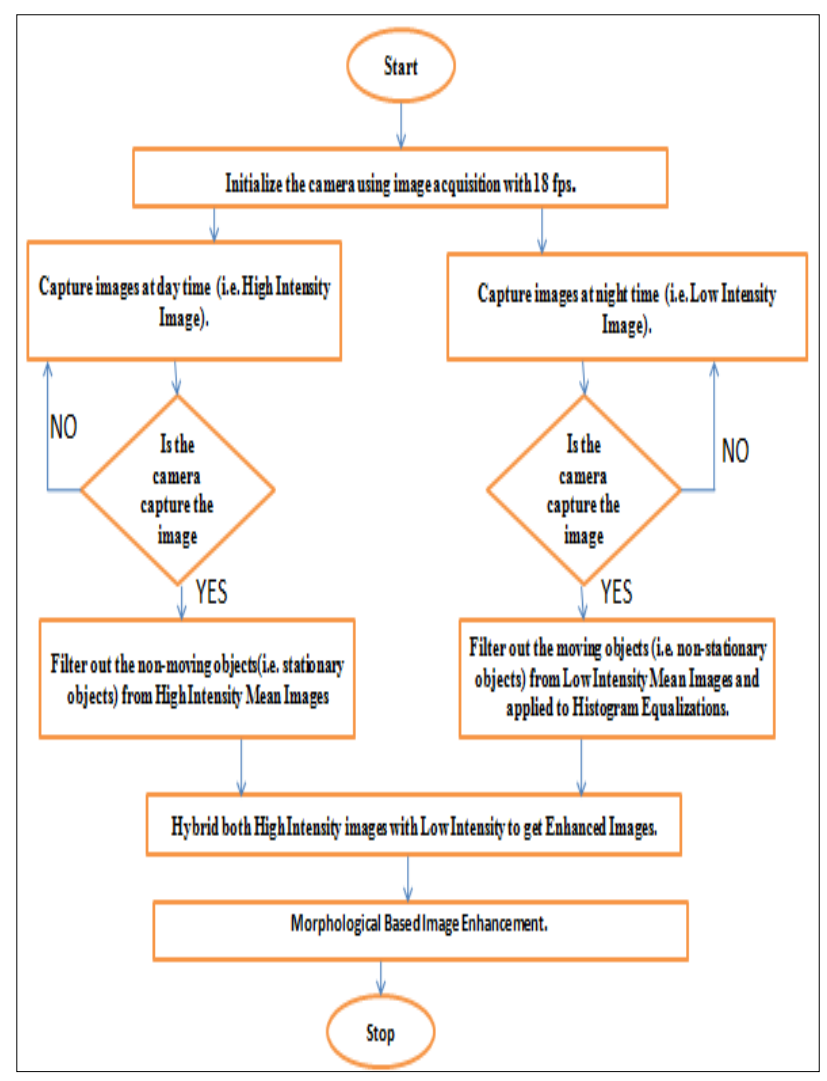

Figure 1: Flow chart of methodology

\subsection{ALGORITHM}

First step: Initialize the camera and Frame/image captured from digital camera with fps more than 18 .

\section{Second step: Hybrid (Day time \& Night time) Technique}

For using the hybrid model for night image enhancement technique, it is important to capture at least five hundred images of local area at day time (i.e. high intensity image) and same for the night time (i.e. low intensity image).

Here we have variables with name $\mathrm{P}=$ Number of pixels of an image (size of every image),

Num= number of images in data base

$\mathrm{DTM}=$ day time matrix (4D matrix to store images in one matrix)

Storing the day time images in one single matrix

$$
\left.\operatorname{DTM}(p, \text { num })=\sum_{n w m=1}^{\text {num }=500} \mathbb{Z}[\operatorname{DTMimg}](1: p, 1)\right]
$$

Now after storing the images for day time in one matrix DTM. We have to take a mean image so that non-moving objects (i.e. stationary objects) extracted in one image DM.

$$
D M=\sum_{n w m=1}^{\text {num=500 }} D T M\left(\mathrm{~s}_{g} \text { num }\right)
$$

Storing the day time images in one single matrix NTM=Night time matrix (4D matrix to store images in one matrix)

$\operatorname{NTM}(p$, num $)=\sum_{\text {num }=1}^{\text {num=500 }}[\operatorname{NTMimg}(1 ; p, 1)]$

Now after storing the images for night time in one matrix NTM. We have to take a mean image so that moving objects (non-stationary objects) extracted in one image NM.

$N M=\sum_{\text {num=1 }}^{\text {num }=500} \operatorname{NTM}(\mathrm{s}$, num $)$

Taking live images with the variable name img where $\mathrm{n}$ is the nth number of image form the starting and subtracting from the night mean image to extract non-stable objects in night.

$$
\begin{aligned}
& D I F F_{P_{r}}=N M_{r}-i m g_{r} \\
& D I F F_{P_{g}}=N M_{g}-i m g_{g} \\
& D I F F_{P_{b}}=N M_{b}-i m g_{b}
\end{aligned}
$$

Removing the noise from DIFF image below 9 threshold value. Storing the dimensions as row and Column of image in Row and Column variables and replacing rest all with day time mean image (DM).

In the next part, have done the fusion of both images i.e. high intensity image with low intensity image to get the enhancement of image.

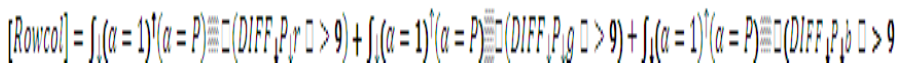

Third step: Calculation of night image enhancement algorithm with threshold variable ' $\alpha$ '.

\section{RESULTS}

In our work of night image enhancement, we take the image of the size $640 x 480$. We consider two metrics to show the experimental results of the work. Metrics are PSNR and MSE. The graphs show that the resultant enhanced image has increased PSNR and decreased MSE as compared to the original night image. 
The graphs showing PSNR and MSE of original night image and enhanced image has been given in this paper for 100 frames. The resultant enhanced image for given night image has also been shown.
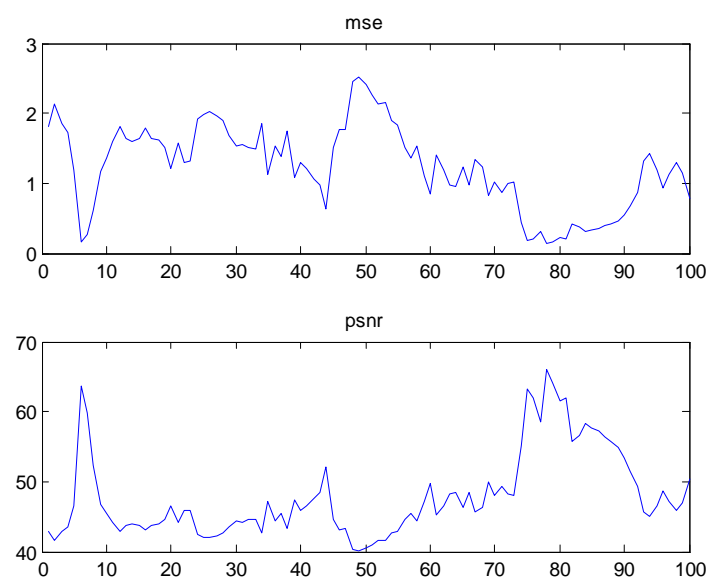

Figure 2: PSNR and MSE after applying fusion technique

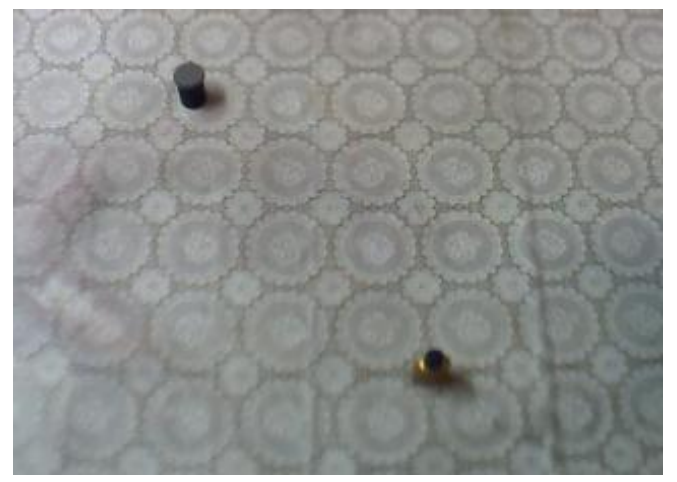

Figure 3. Day Time Image

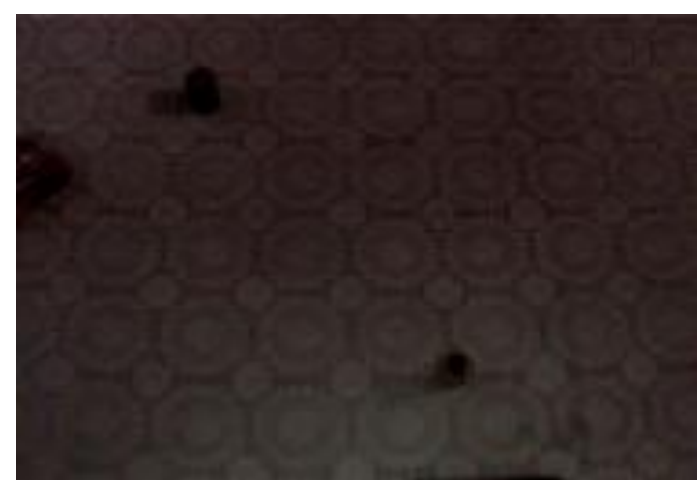

Figure 4. Night Time Image

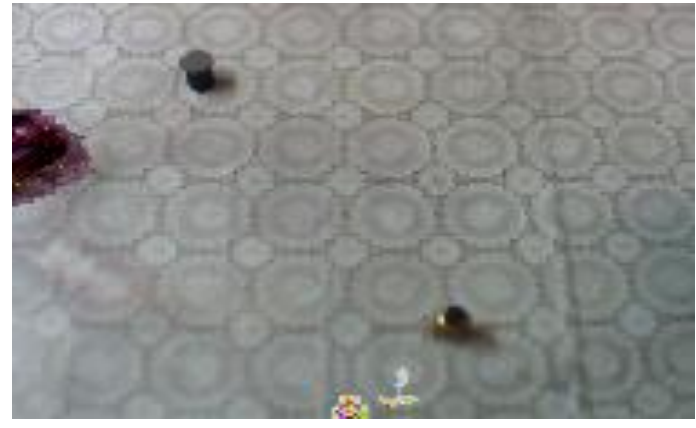

Figure5: Enhanced Image Using Fusion Technique

Figure 3 represents that image is captured at day time. In this image two corks are used as stationary objects.

Figure 4 represents that image is captured at night time. In this image two corks are used as stationary objects and car is as moving object.

Figure 5 represents that image is enhanced with the use of fusion technique. In this image, the car is taken from night image and background image which have two corks as stationary objects taken from day time image. Both of these images are fused to get the enhanced image.

\section{CONCLUSION}

Night image enhancement techniques are widely applicable to different fields. The proposed method has given good results in terms of MSE and PSNR. This algorithm can be used even in darkness. By changing the threshold values different views and different objects can be seen for same image by the users. The noise has also been properly removed from the images. The resultant enhanced image closely resembles the day image by using this algorithm.

\section{ACKNOWLEDGEMENTS}

The authors are thankful to Dhiraj Kumar Singh and Gagandeep Singh, Assistant Professor, Rayat Institute of Engineering \& Information Technology, Railmajra for providing technical supports during preparation of the manuscript.

\section{REFERENCES}

[[1] Vinay G. Vaidya, Chandrashekhar N. Padole "Night Vision Enhancement Using Wigner Distribution”, IEEE 2008.

[2] Xiaoying Fang, Jingao Liu, Wenquan Gu, Yiwen Tang "A Method to Improve the Image Enhancement Result based on Image Fusion",IEEE 2011.

[3] Li, G. and Wang, K., Applying daytime colours to nighttime imagery with an efficient colour transfer method, In: J.G. Verly \& J.1. Guell (Ed.), Enhanced and Synthetic Vision 
2007, pp. 65590L-1-65590L-12, The International Society for Optical Engineering, Bellingham, MA, 2007.

[4] Shi, J., Jin, W., Wang, L. and Chen, H., Objective evaluation of colour fusion of visual and IR imagery by measuring image contrast, In: H. Gong, Y. Cai \& J.-P. Chatard (Ed.), Infrared Components and Their Applications, pp. 594601, The International Society for Optical Engineering, Bellingham, MA, 2005.

[5] Shi, 1.-S., Jin, W.-Q. and Wang, L.-X., Study on perceptual evaluation of fused image quality for colour night vision, Journal ofInfrared and Millimeter Waves, 24(3) ,pp. 236-240,2005.

[6] Tsagaris, V. and Anastasopoulos, D., Multispectral image fusion for improved RGB representation based on perceptual attributes, International Journal of Remote Sensing, 26(15) ,pp. 3241-3254, 2006.

[7] Zheng, Y., Hansen, B.C., Haun, A.M. and Essock, E.A., Colouring night-vision imagery with statistical properties of natural colours by using image segmentation and histogram matching, In: R. Eschbach \& G.G. Marcu (Ed.), Colour imaging $\mathrm{X}$ : processing, hardcopy and applications, pp. 107117, The International Society for Optical Engineering, Bellingham, WA, 2005.

[8] Ramesh Raskar,Adrian llie and Jingyi Yu, "Image Fusion for Context Enhancement and video surrealism" ,The 3rd International Symposium on Non-Photorealistic Animation and Rendering (NPAR), Annecy, France, 2004.

[9] I.Li, S. Z.Li, Q. Pan, and T. Yang "111umination and Motion-Based Video Enhancement for Night Surveillance" In Proc. of the 2nd Joint IEEE International Workshop on YSPETS,pages 169-175. Beijing, China, October 2005.

[10] Akito Yamasaki, Hidenori Takauji, Shun' ichi Kaneko, "Denighting: Enhancement of Nighttime Images for a Surveillance Camera", Kita 14, Nishi 9, Kita-ku, Sapporo 0600814, JAPAN.

[11] Manpreet Kaur, Sukhwinder Singh "Night Image Enhancement using Hybrid of Good and Poor Images" IJCTA, July-August 2012.

\section{BIOGRAPHIES:}

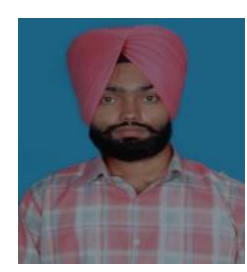

Amritpal Singh received the B.Tech degree in Electronics and Communication Engineering from Punjab Technical University, Jallandhar. Presently, he is working as Lecturer in RIEIT, Railmajra as well as pursuing M.Tech in ECE from ACET, Amritsar. His current research interests include Image Processing.

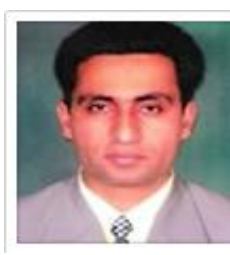

Dr. Vijay Kumar Banga received the B.Tech degree in Electronics and Communication engineering from Punjabi University Patiala, M.Tech degree in Electronics and Communication Engineering from Panjab University Chandigarh and Phd. From Thapar
University Patiala. $\mathrm{He}$ is currently working as HOD in Amrisar College of Engineering and Technology, Amritsar. His current research interests include Soft Computing, Image Processing, Autonomous Robots. 